\title{
Health Condition Alarm System
}

\author{
Maiga Chang ${ }^{1}$, Ebenezer Aggrey ${ }^{1}$, Mehadi Sayed ${ }^{2}$, and Kinshuk ${ }^{1}$ \\ ${ }^{1}$ School of Computing and Information Systems, Athabasca University, Canada \\ maiga@ms2.hinet.net, aggreyeb@shaw.ca,

\begin{abstract}
A Health Condition Alarm System has been developed to provide practitioners an update on their patients' conditions in real-time. This paper focuses on how researchers and doctors can use the system to create vital sign ranges and alarm levels as well as to see the alarm alerts. The system is webbased and open-access, and the research team would like to collaborate with practitioners, hospitals, laboratories, and medical professionals to expand the system's application domains. The research team has leveraged two quality control methods, X-Chart and Westgard Multi-Rule, to decide the alarm level of a patient's health condition and the alarm timing. Four alarm levels (i.e., normal, subnormal, cautious, and alert) can then be decided by the mean values and standard deviations of the patient examination data.
\end{abstract}

Keywords: Healthcare, Data Mining, Westgard Multi-Rule Quality Management, X-Chart, Levey-Jennings Control Chart.

\section{Introduction}

The research team intended to design an ideal tele-healthcare system for elderly healthcare patients [1-3]. With this system's help, doctors can obtain a clear picture of the health conditions of elderly patients without the patients being required to visit the hospital frequently. The research team used X-chart and Westgard multi-rule quality management [4-5] to first analyze patients' physical examination data and then categorize their health conditions into different alarm levels. These alarm levels remind medical professionals when to take action and also reduce the chance of false alarm being generated [6]. The algorithm was implemented as a built-in module of the telehealthcare system; outside the tele-healthcare system, the module cannot be accessed. Additionally, the vital signs, vital sign ranges, and alarm rules are fixed and cannot be altered without recompiling the entire system.

Because the criteria of health condition measurements vary among different countries, regions, and healthcare professionals, the research team implemented a more sophisticated and flexible open-access health condition alarm system on the Internet. The system itself is a complete solution that health practitioners working in clinics can use it to enter patient's examination data and their observations, diagnosis, and suggestions. Furthermore, the system can also be extended to support complicated lab data

K. Imamura et al. (Eds.): BHI 2013, LNAI 8211, pp. 307-315, 2013.

(C) Springer International Publishing Switzerland 2013 
results feeds and corresponding rules establishment, for instances, the normal body temperature ranges from $97.6 \mathrm{~F}$ to $99.6 \mathrm{~F}$; the adult fever temperature ranges from 100 $\mathrm{F}$ (oral temperature) to $101 \mathrm{~F}$ (rectal or ear temperature); and, the child fever temperature ranges from $100 \mathrm{~F}$ (oral temperature) to $100.4 \mathrm{~F}$ (rectal or ear temperature).

This paper is organized into three main sections. Section 2 explains the fundamental concepts and designs of the health condition alarm mechanism. (For more details on this topic, readers can study the research team's previously published work [6].) Section 3 discusses introduces how health practitioners can use the system. Last, section 4 demonstrates the flexibility of the system and explains how medical professionals can set up and add health conditions, lab data units and ranges, as well as rubric information.

\section{Concepts of the Health Condition Alarm Mechanism}

It is important to provide healthcare practitioners with an update or notification of a patient's health condition (assessed and diagnosed through examination data, e.g., vital signs and lab results). This research uses two quality control methods, X-Chart and Westgard Multi-Rule, to decide the specific alarm level that corresponds to a patient's health condition and the alarm timing. An alarm has four levels: normal (A), subnormal (B), cautious (C), and alert (D). Alarm levels are decided by the X-Chart method based on the mean values (M) and standard deviations (SD) of the patient examination data [7].

After acquiring the mean value and standard deviation of the examination data, the top limit is set as $\mathrm{M}+3 \mathrm{SD}$ and the bottom limit is set as M - 3SD. The data in the range between $\mathrm{M}+3 \mathrm{SD}$ and $\mathrm{M}-3 \mathrm{SD}$ is called the action limit, as Figure 1 shows. If the patient's examination data goes beyond the action limit, then the Westgard MultiRule method may be applied to reassess the patient's examination data. Data that falls into the range between $\mathrm{M}+2 \mathrm{SD}$ and $\mathrm{M}-2 \mathrm{SD}$ will be seen as an "Alert."

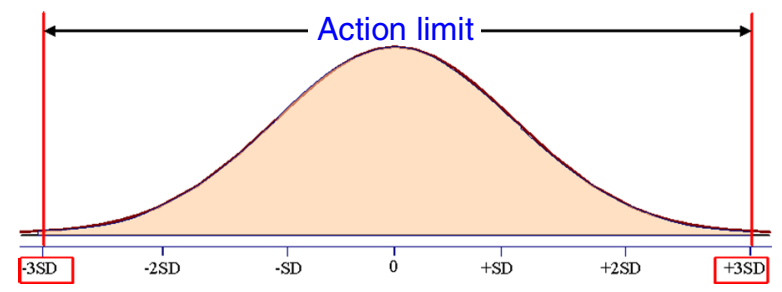

Fig. 1. Action Limit

The range between $\mathrm{M}+2 \mathrm{SD}$ and $\mathrm{M}-2 \mathrm{SD}$ is the warning limit (Figure 2). If the patient's examination data goes beyond the warning limit, then practitioners may need to take appropriate actions for the patient. The warning limit includes three alarms levels: normal (A), subnormal (B), and cautious (C). The range between $\mathrm{M}+1.5 \mathrm{SD}$ and $\mathrm{M}+2 \mathrm{SD}$ or between $\mathrm{M}-1.5 \mathrm{SD}$ and $\mathrm{M}-2 \mathrm{SD}$ is "cautious"; the range between $\mathrm{M}$ $+\mathrm{SD}$ and $\mathrm{M}+1.5 \mathrm{SD}$ or between $\mathrm{M}-\mathrm{SD}$ and $\mathrm{M}-1.5 \mathrm{SD}$ is "subnormal"; and the range between $\mathrm{M}+\mathrm{SD}$ and $\mathrm{M}-\mathrm{SD}$ is "normal." 


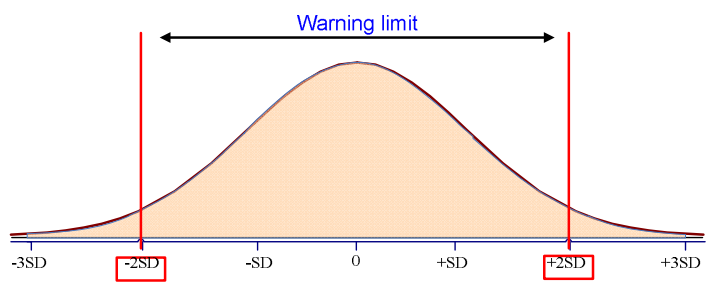

Fig. 2. Warning Limit

\section{The Health Condition Alarm System for Practitioners}

The research team is aiming to make the health condition alarm mechanism available to all healthcare practitioners, as well as hospitals, healthcare facilities, and medical service providers; hence, a web-based open-access solution is the most expedient option. We would also like to provide healthcare practitioners and facilities a complete and flexible health condition alarm solution; therefore, we design and implement all functions that doctors and administrative professionals require. In this paper, we will focus on the use cases of doctors and administrative professionals who have used the system on a trial basis.

As shown by Figure 3, the Health Condition Alarm System is comprised of a number of independent components. These components were individually developed in Java; as the project progressed, the components were integrated with one another when appropriate. The system is running on the Linux server (i.e., Debian) including all other GNU General Public License servers and services. Since one of our objectives is to offer doctors and administrative professionals a complete, free, open-access online solution, the system has two pre-determined user roles: doctor and administrator.

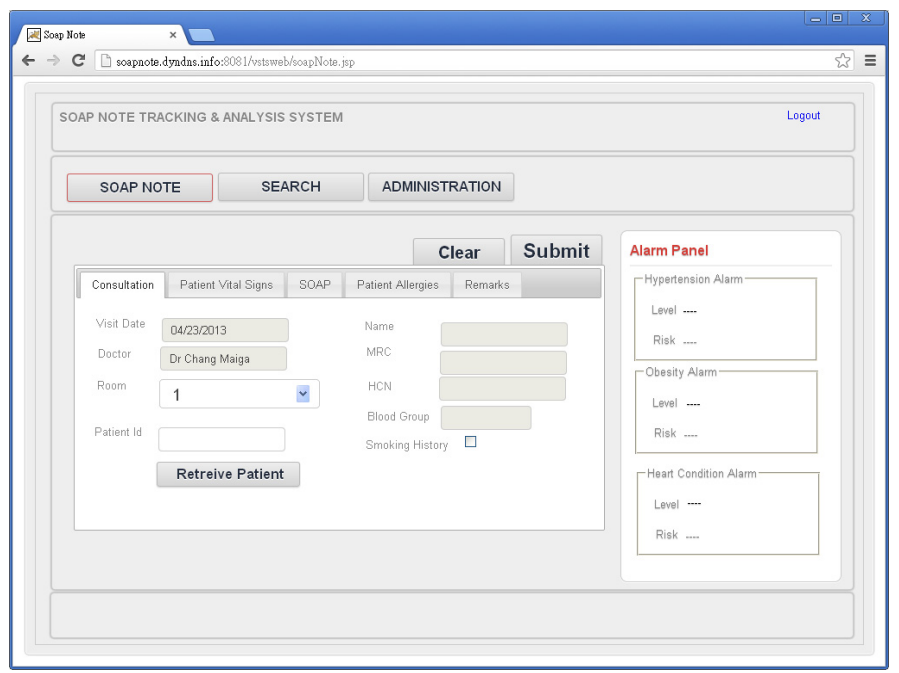

Fig. 3. Main screen of the system 
When a practitioner signs in to the system, there will be three main functions or tasks the practitioner can access and perform, shown in Figure 3. However, before the practitioner can see the heads-up notification of a patient's health condition, he or she must access the patient's data by entering the patient's ID and clicking the "Retrieve Patient" button.

The practitioner can enter examination data as well as his or her diagnosis, comments, and suggestions for the patient (see Figure 4). Once the data and accompanying notes have been entered into the system, the health condition alarm mechanism will be activated. For example, a practitioner can see a subnormal notice with the risk of getting into pre-hypertension stage on the alarm panel at the right hand side of the screen (Figure 5). A practitioner can also choose whether or not to see the alarms whose levels are not over a certain level.

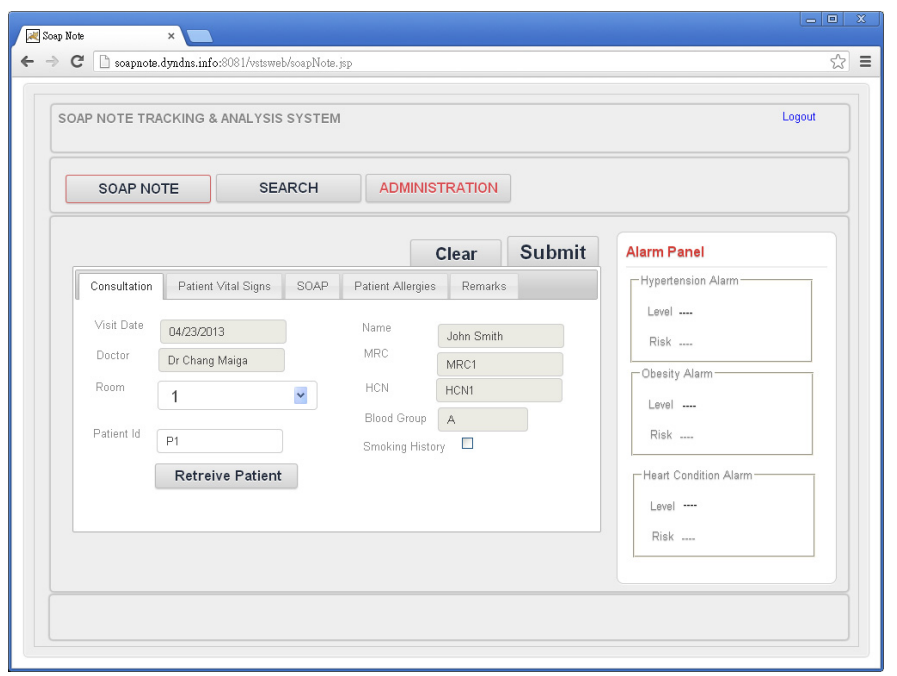

Fig. 4. Practitioners can enter examination data, analysis results and comments after retrieving the patient's information

If the practitioner needs to, he or she can also search the historical data for the patient. When this is done, the health condition alarm mechanism is automatically applied to the time period of the historical data; the practitioner can then see notifications regarding the patient's health condition (Figure 6). In this simulation case, the practitioner can see two subnormal notices relating to hypertension and obesity problems the patient has.

\section{$4 \quad$ Health Condition Alarm System for Administrative Users}

Since the criteria for measuring and evaluating a patient's health condition(s) through the analysis of examination data and lab results differ among health facilities around the world, the system provides administrative professionals with functions to: (1) add labels (i.e., vital signs in the system), units, and ranges for lab results; (2) add health 


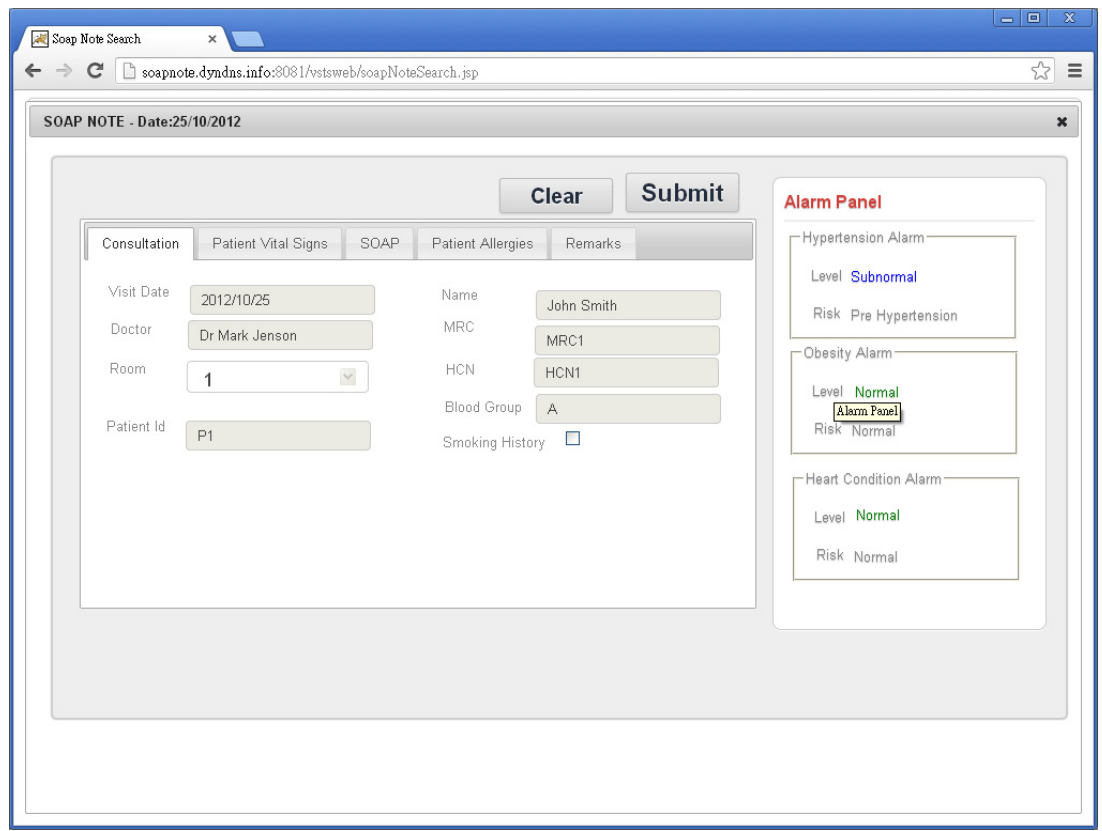

Fig. 5. Practitioners can see the alarm(s) once new data has been entered.

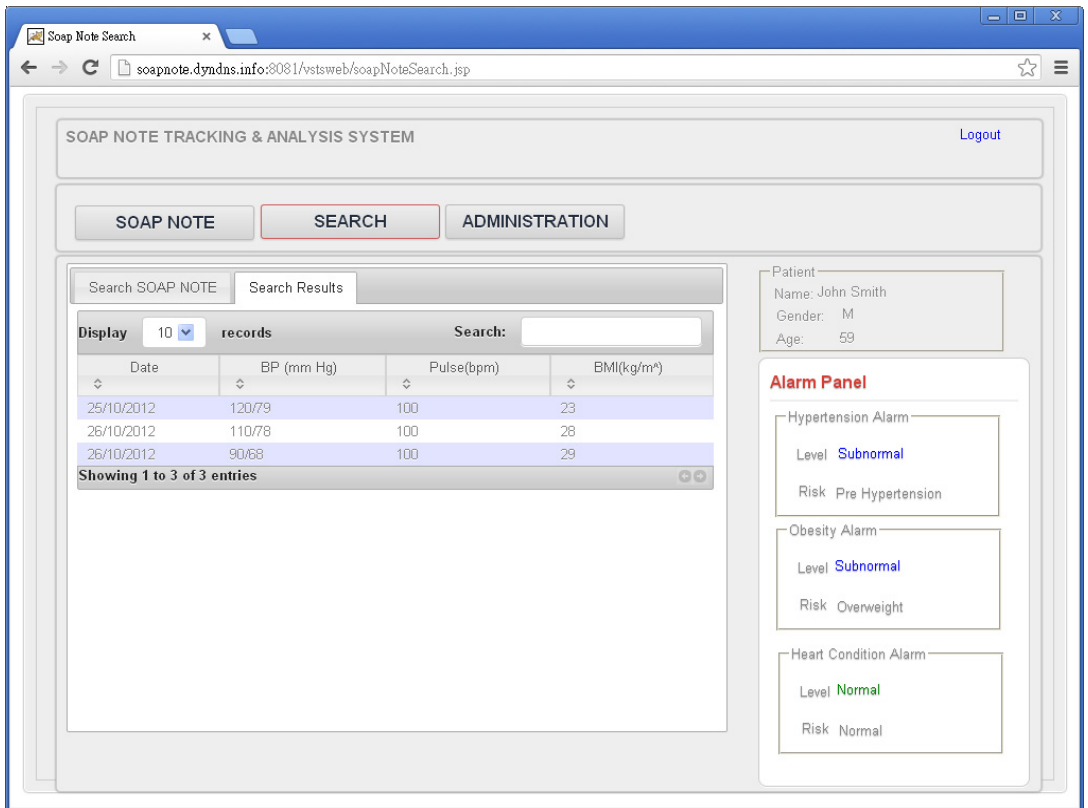

Fig. 6. Practitioners can search the historical data of his or her patient and the health condition alarm mechanism is applied to the historical data automatically 
conditions and manage the associations between the health conditions and vital signs; and (3) establish the connections among alarms and health condition risks.

If administrative professionals have any lab results which the current system does not support, they can use the Vital Sign Management function (shown in Figure 7) to add new vital sign names and their units. Once vital signs have been added, practitioners can enter the data or information for the patient(s). Because the alarm mechanism uses mean values and standard deviations to test the examination data, the administrative professionals need to add ranges for the vital signs (see Figure 8).

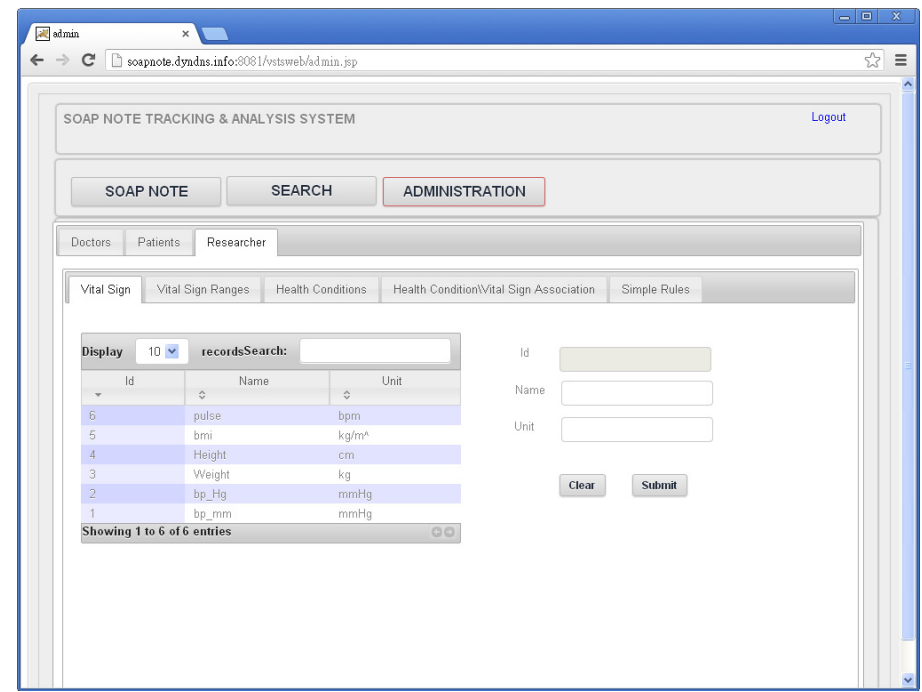

Fig. 7. Administrative professionals can add new vital sign names for lab results

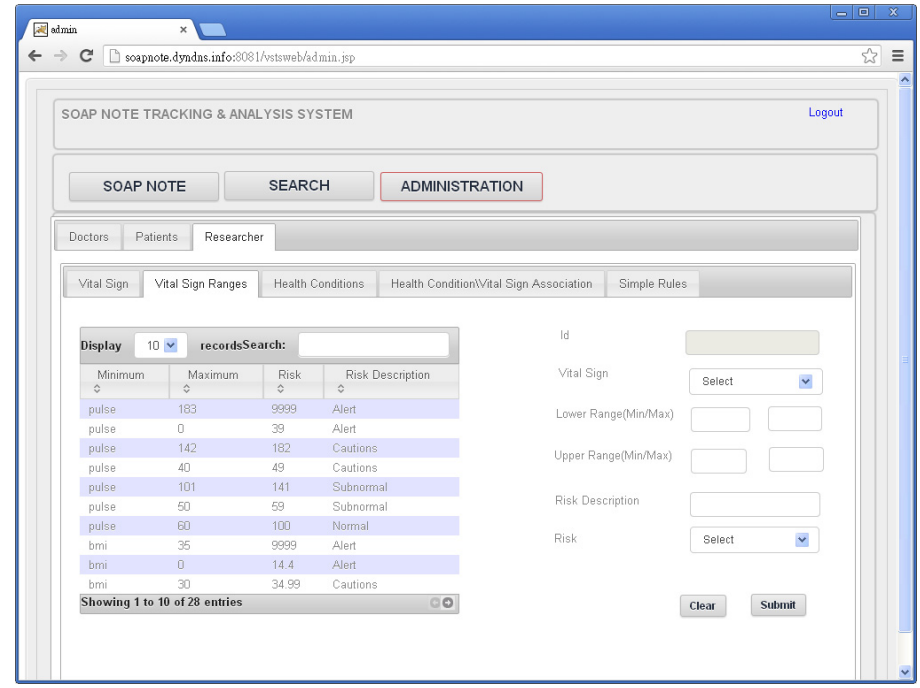

Fig. 8. Administrative professionals can add alarm ranges for particular vital signs 
The assessment of a health condition can only be made based on one or more vital signs. For example, the practitioner may need to consider both blood pressure and pulse to tell if a patient is at risk for hypertension problems. In this system, the administrative professionals can first add the health condition(s) for the newly added vital signs (shown in Figure 9). They can then relate the vital signs (i.e., lab results) with the health condition(s), as Figure 10 shows.

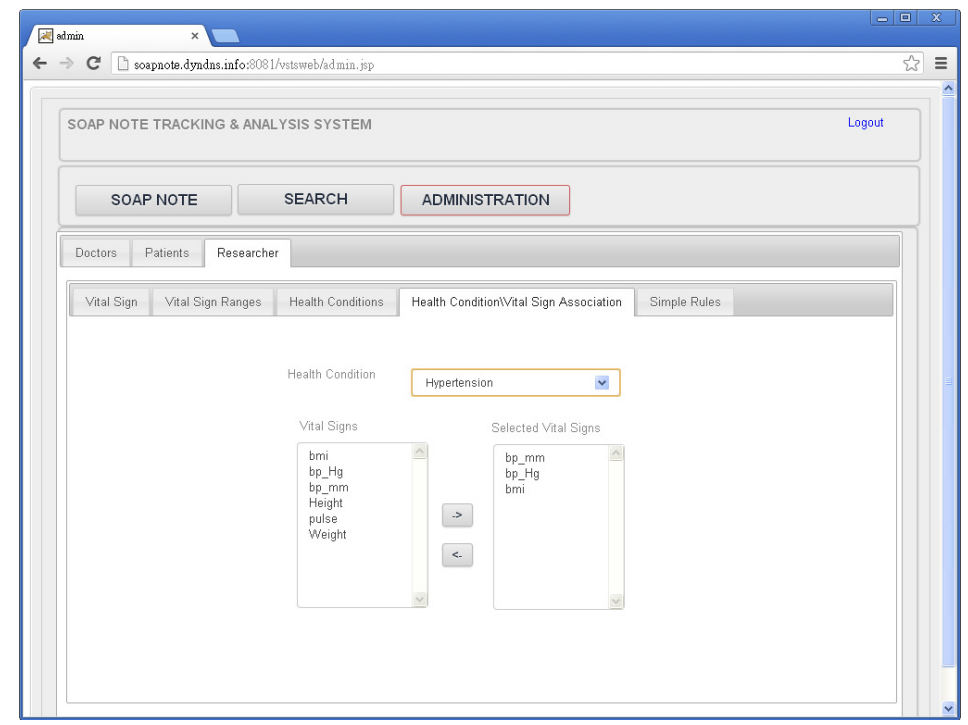

Fig. 9. Administrative professionals can add health condition(s) for particular vital signs

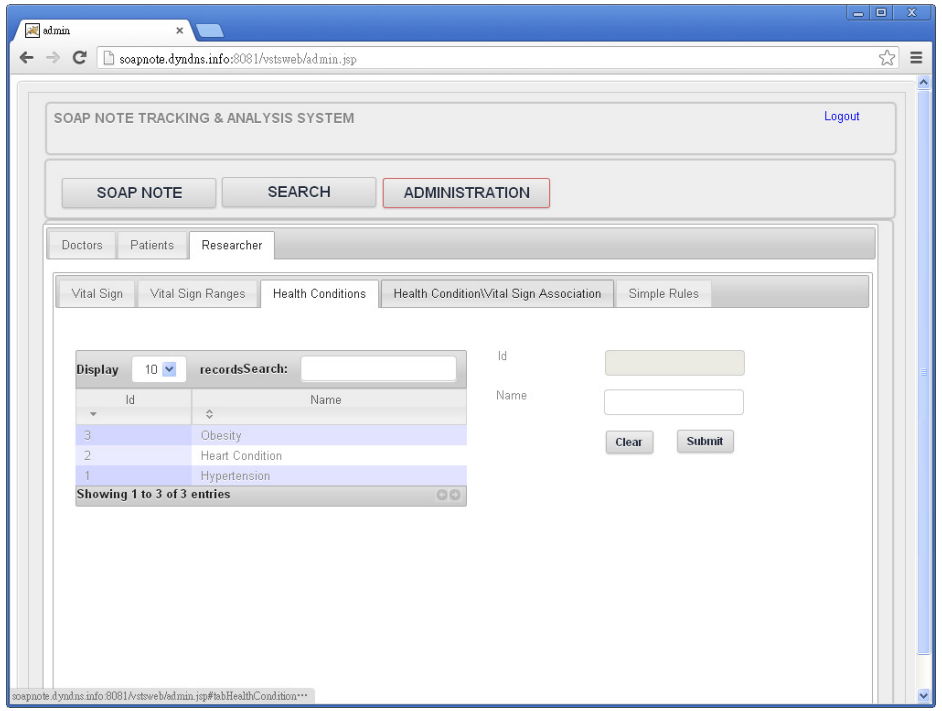

Fig. 10. Administrative professionals can associate health condition(s) with vital signs 
The third function administrative professionals can perform deals with creating a rubric or set of rules which link both the alarm levels of vital signs and the risks of getting health problems together. For instance, a patient may be in one of four risk categories of having hypertension: normal, pre-hypertension, hypertension stage 1, and hypertension stage 2. Administrators thus need the Rules function (shown in Figure 11) to create the rubric, so that healthcare practitioners can see the appropriate alarm-relevant notifications in the alarm panel.

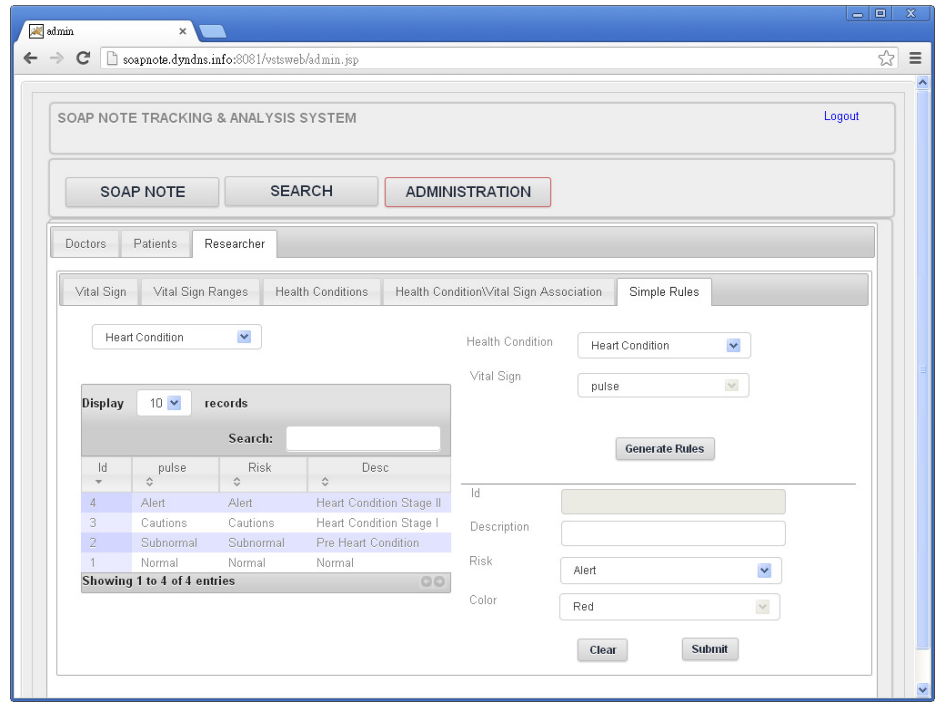

Fig. 11. Administrative professionals can create rules for specific health problem

\section{Conclusion}

The Health Condition Alarm System is a web-based open-access system for health practitioners, healthcare organizations and facilities such as hospitals and clinics, and medical professionals. The system is self-sustainable and has all the functions that practitioners and administrative professionals need: The system not only allows administrative professionals to add new lab results, health conditions and risks, but also allows them to customize (depending on country and/or different facility) using their own criteria of measuring a patient's health condition(s) in regards to reading and analysing lab results. Individual patients can also use the system to both monitor their health condition(s) and receive alarms or notifications about the likelihood and risks of having health problems. All that is required to use the system is a record of their examinations data and lab results.

The research team would like to collaborate with healthcare practitioners, clinics, hospitals, organizations, and information service providers (i.e., industry partners) to test the usability of this system as well as to get user perceptions and comments about the system to make further improvements in the future. The research team also hopes 
to have the opportunity to expand the database of vital signs and health conditions, so that the system can be more beneficial and effective for users.

Acknowledgements. The authors wish to thank NSERC, iCORE, and Xerox for their support. We also could not have done the project without the research-related funding generously gifted to the Learning Communities Project by Mr. Allan Markin.

\section{References}

1. Kahn, S., Sheshadri, V.: Medical Record Privacy and Security in a Digital Environment. IEEE IT Professional 10(2), 46-52 (2008)

2. Maglaveras, N., Chouvarda, I., Koutkias, V.G., Gogou, G., Lekka, I., Goulis, D., Avramidis, A., Karvounis, C., Louridas, G., Balas, E.A.: The Citizen Health System (CHS): A Modular Medical Contact Center Providing Quality Telemedicine Services. IEEE Transactions of Information Technology in Biomedicine 9(3), 353-362 (2005)

3. Stantchev, V., Schulz, T., Hoang, T.D., Ratchinski, I.: Optimizing Clinical Processes with Position-Sensing. IEEE IT Professional 10(2), 31-37 (2008)

4. Westgard, J.O., Barry, P.L., Hunt, M.R., Groth, T.: A multi-rule Shewhart chart for quality control in clinical chemistry. Clinical Chemistry 27(3), 493-501 (1981)

5. Westgard, J.O., Barry, P.L.: Improving Quality Control by use of Multirule Control Procedures. In: Westgard, J.O., Barry, P.L. (eds.) Cost-Effective Quality Control: Managing the Quality and Productivity of Analytical Processes, pp. 92-117. AACC Press, Washington (1986)

6. Chang, M., Heh, J.-S., Lin, H.-N.: Tele-Physical Examination and Tele-Care Systems for Elderly People. Journal of Community Informatics 8(1) (2012),

http: / / ci-journal.net/index.php/ciej/article/view/762/900 (retrieved)

7. Tseng, C.-H., Lin, H.-N., Cheng, S.-Y., Heh, J.-S., Lo, W.-M.: Designing a Clinical Alert Mechanism Based on X-Chart in Physical Signal Examination System. In: 14th International Congress of Oriental Medicine, Taipei, Taiwan (2007) 